\title{
Multilevel Embedding of multiple images with DWT, DCT and SVD
}

\author{
${ }^{1}$ Maruturi Haribabu, ${ }^{2} \mathrm{Ch}$. Hima Bindu \\ ${ }^{1}$ Assistant Professor, ECE Department, QISCET, ongole, Andhra Pradesh, India, ${ }^{2}$ ECE Department , QISCET, ongole, \\ Andhra Pradesh, India \\ Email: ${ }^{1}$ haribabu.maruturi@gmail.com, ${ }^{2}$ hb.muvvala@gmail.com
}

\section{Received: $18^{\text {th }}$ February 2018, Accepted $17^{\text {th }}$ March 18, Published: $30^{\text {th }}$ April 2018}

\begin{abstract}
In this paper, Discrete Wavelet Transform (DWT), discrete cosine Transform (DCT) and Singular Value Decomposition (SVD) based Image watermarking scheme. The idea of proposed method is multiple hidden images watermarking technique that means using two cover images and three watermark images. Initially the three watermark images are embedding into second cover image to get watermarked image and again the resultant watermarked image is embedding into first cover image after this embedding process finally obtained final watermarked image. At the receiver side perform reverse operation we can get three watermark images. This proposed method is superior to existing method. This method is robust to noise attacks and imperceptible and also improvement of experimental results than the existing method related to Peak Signal to Noise Ratio (PSNR), Mean Square Error (MSE) and Root Mean Square Error (RMSE)
\end{abstract}

Keywords: Discrete Wavelet Transform (DWT), discrete cosine Transform (DCT), Singular Value Decomposition (SVD), Digital Image Watermarking, PSNR, MSE and RMSE.

\section{INTRODUCTION}

From the last decays the growth and usage of internet is very high but security is the major issue from unauthorized persons. To improve of security level in network for sharing and disturbing of data from unauthorized persons using digital image watermarking. Digital image watermarking is the process of Digital image watermarking is categorized into two processes: Firstly process of maintain the secret image (watermark mark image) into host image i.e. watermarked image. This process is called embedding process. At the receiver side perform the reverse operation to extract original secret image i.e. Extraction process [1].

The Digital image watermarking performed into either spatial domain or frequency domain. In spatial domain directly perform the modification of image pixel values. The best example of spatial domain technique in digital image watermarking is LSB (least significant bit). The spatial domain technique is the less complexity and shortest processing time but less robustness. To improve the robustness using transforms domain techniques or methods. Initially in transform domain (frequency domain) perform the watermarking by the modification of co-efficient values. The well known transform domain methods are Discrete Wavelet Transform (DWT) [2,3], discrete cosine Transform (DCT) [4], Singular Value
Decomposition (SVD) [5], DFT (Discrete Fourier transform) [5] etc.

This paper demonstrates the watermarking scheme based on Discrete Wavelet Transform (DWT) [2,3], discrete cosine Transform (DCT) [4], Singular Value Decomposition (SVD) [5]. The main aim of this paper is to improve the enough robustness against different noise attacks and also imperceptibility [1].

The outline of this paper discuss is given below: In section II discuss about the related transform domain techniques, In section III describes the proposed method and finally Experimental results and conclusion in section IV and V.

\section{Preliminaries}

DWT (Discrete Wavelet transform) [2,3] is one of the mathematical tools to represents an image in to different number of resolutions. One level DWT, the entire image decomposed into four coefficient values i.e. LL band, LH band, HL band \& $\mathrm{HH}$ band. It can be further down sampling by two levels DWT and more. To obtained original image after inverse DWT process

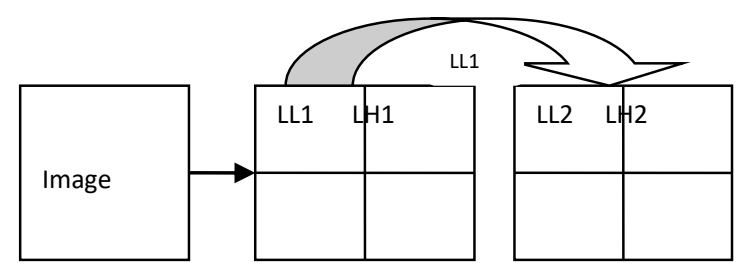

Fig 1. Multi level DWT

SVD (Singular Value Decomposition) [5] is a one of the image watermarking technique to improve the security. This is one of the methods to extract the statistical properties of the image. Let us consider the image $A$, SVD of $A$ generates two singular matrices $U$, and $V$ and one diagonal matrix $S$.

$$
S V D(A)=U S V^{T}
$$


DCT (Discrete Cosine Transform) is another important frequency domain technique. It is mainly applicable for Image compression, Image watermarking [4]

$$
\begin{gathered}
F(P, Q)=\alpha(P) \alpha(Q) \sum_{x=0}^{X-1 Y-1} \sum_{y=0} f(x, y) \cos \left[\frac{(2 x+1) \pi P}{2 X}\right] \cos \left[\frac{(2 y+1) \pi Q}{2 Y}\right] \\
f(x, y)=\sum_{P=0 Q}^{X-1 Y-1} \alpha(P) \alpha(Q) F(P, Q) \cos \left[\frac{(2 x+1) \pi P}{2 X}\right] \cos \left[\frac{(2 y+1) \pi Q}{2 Y}\right] \\
\alpha(P)= \begin{cases}\sqrt{\frac{1}{X}} & \text { if } P=0 \\
\sqrt{\frac{2}{X}} & \text { if } P \neq 0\end{cases} \\
\alpha(Q)= \begin{cases}\sqrt{\frac{1}{Y}} & \text { if } Q=0 \\
\sqrt{\frac{2}{Y}} & \text { if } Q \neq 0\end{cases}
\end{gathered}
$$

\section{Proposed MeThod}

To protect the data from any unauthorized person using image watermarking. The digital image watermarking is a one of important research area to provide the security. The main specialty of image watermarking is to embed the data in host image using scaling factor. The proposed embedding algorithm is it imperceptible and should have high robustness. There are different ways to embedding the watermark into host image using in frequency domain.

\section{A. Watermark hiding process}

\section{$>$ Level 1 Embedding Process:}

Step1: Read the color cover images in1 $(512 * 512 * 3)$, in2 $(256 * 256 * 3)$ and three gray level watermark images wa1 (256*256), wa2 (256*256) and wa3 (256*256).

The process of initial watermark embedding process is as follows:

Step 2: Apply SVD on three components (Red, Green and blue) of second cover image in 2 to get three different $\mathrm{u}, \mathrm{v}$ $\&$ s matrices (or) co-efficient.

$$
\left[u_{i} s_{i} v_{i}\right]=\operatorname{svd}\left(\operatorname{in} 2_{i}\right) \quad \mathrm{i}=1,2,3
$$

Step 3: Apply SVD on three watermark images (wa1, wa2 \&wa3) to get three different $\mathrm{u}, \mathrm{v} \& \mathrm{~s}$ matrices (or) coefficient.

$$
\left[u w a_{i 1} s w a_{i 1} v w a_{i 1}\right]=s v d\left(i n 2_{i 1}\right) \quad \mathrm{i} 1=1,2,3
$$

Step 4: The singular values of three watermark images (swa1, swa2 \& swa3) embedding into singular values of red, green and blue components of second cover image in2 (s1, s2 \& s3) with key (or) scaling factor $\alpha$ to obtain new singular values nsi.

$$
n s i=s_{i}+\left(\alpha * s w a_{i 1}\right)
$$

$$
\begin{gathered}
\alpha=\frac{1}{\mu} \\
\text { Where } \quad \mu=\frac{1}{X \times Y} \sum_{x=0}^{X-1} \sum_{y=0}^{Y-1} i n 2_{1}(x, y)
\end{gathered}
$$

Step 5: After embedding process performs inverse SVD we can get three new red, green and blue components using the below formula

$$
n e w_{i}=u_{i} \times n s_{i} \times v_{i}
$$

Step 6: finally initial watermarked image (wd1) can be obtained by concatenation of three new red, green and blue components.

Level 2 Embedding Process:

Step 7: Apply one level DWT on blue component of first cover image in1 to get four coefficients ( $\mathrm{LL}, \quad \mathrm{LH}, \mathrm{HL}$ \& $\mathrm{HH}$ ) and also perform DCT on LH,HL,HH coefficients to obtain three DCT coefficient values (dc1, dc2 \& dc3).

Step 8: The red (nrwd1), green (ngwd1) \& blue (nbwd1) components of initial watermarked image (wd1) is embedding into DCT coefficients of cover image in1 with scaling factor $\alpha 1$ i.e. 0.005 .

$$
\begin{aligned}
& \operatorname{ndc} 1=\operatorname{dc} 1+(\alpha 1 * \text { nrwd } 1) \\
& \operatorname{ndc} 2=\operatorname{dc} 2+(\alpha 1 * \text { ngwd } 1) \\
& \text { ndc } 3=\operatorname{dc} 3+(\alpha 1 * \text { nbwd } 1)
\end{aligned}
$$

Step 9: After this process perform IDCT \& IDWT to get new blue component.

Step10: Finally watermarked image (WD) with concatenation of new blue component with unmodified red and green components respectively.

\section{B. Watermark Retrieve process}

\section{Extraction of Level 2 Process:}

Step 11: Perform the one level DWT on blue component of color watermarked image (WD) to get four coefficients (LLWD, LHWD, HLWD \& HHWD).

Step 12: After DWT, perform DCT on LHWD, HLWD \& HHWD coefficients to obtain new DCT coefficient values (WDdc1, WDdc2 \& WDdc3).

Step 13: To extract Initial watermarked image by using below equations

$$
\begin{aligned}
& r w d 1=\frac{(W D d c 1-d c 1)}{\alpha_{1}} \\
& g w d 1=\frac{(W D d c 2-d c 2)}{\alpha_{1}} \\
& b w d 1=\frac{(W D d c 3-d c 3)}{\alpha_{1}}
\end{aligned}
$$


Step 14: Finally combine above three components (rwd1, gwd1 \& bwd1) to get initial watermarked image (wd1).

Extraction of Level 1 Process:
Step 15: Perform SVD on red, green and blue components of initial watermarked image (wd1) to get three different USV matrices (or) coefficients.

$$
\left[u u_{i} s s_{i} v v_{i}\right]=s v d\left(w d 1_{i}\right)
$$

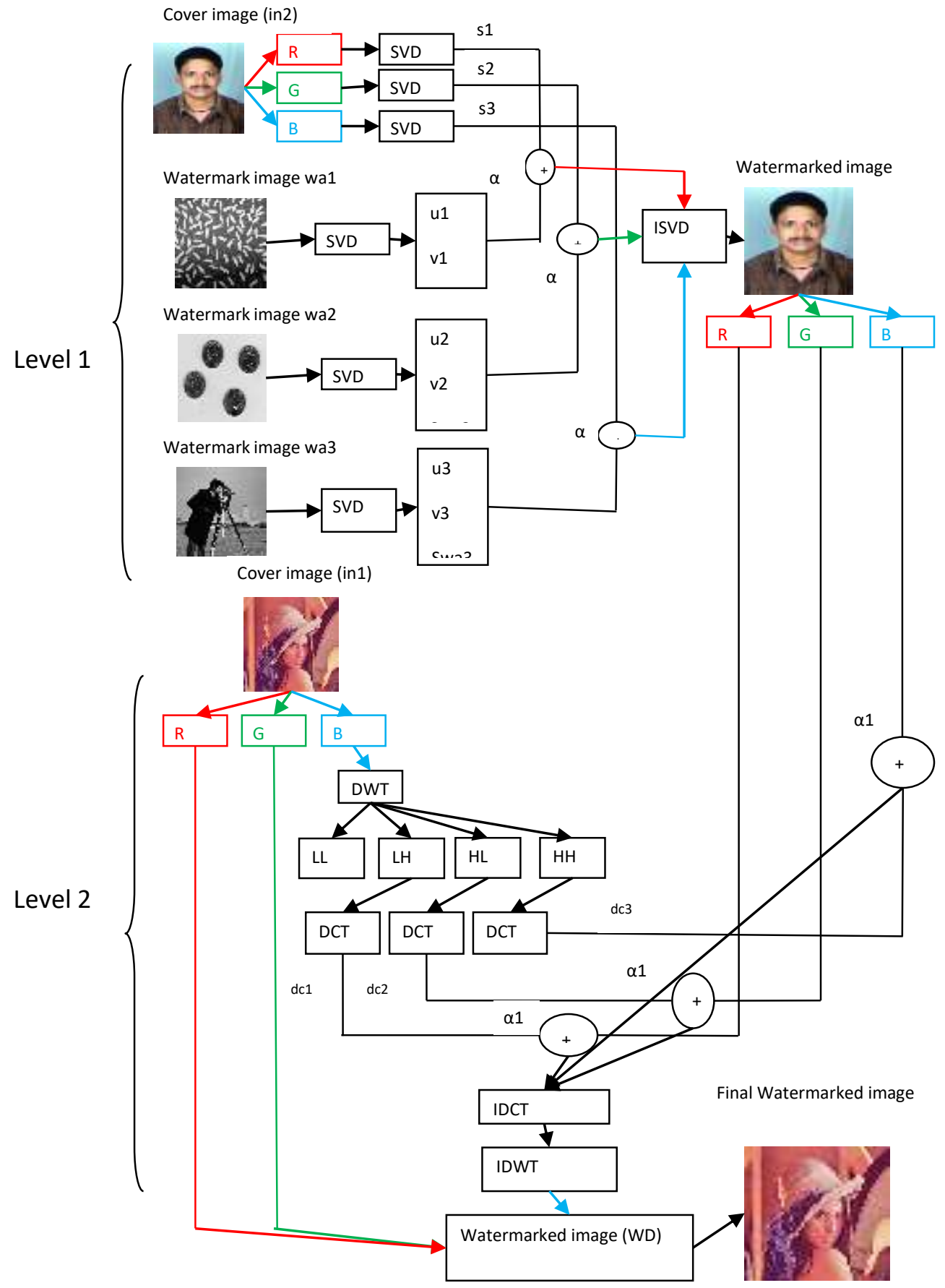

Fig. 2. Flow chart of watermark embedding process 
Step 16: To extract singular values of original watermark images (wa1, wa 2 \& wa3) by using below equations:

$$
n s e_{i}=\frac{\left(s s_{i}-s_{i}\right)}{\alpha}
$$

Step 17: Finally to obtain three original watermark images (wa1, wa2 \& wa3) after performing ISVD of $n s e_{i}$ with $u w a_{i} \& v w a_{i}$

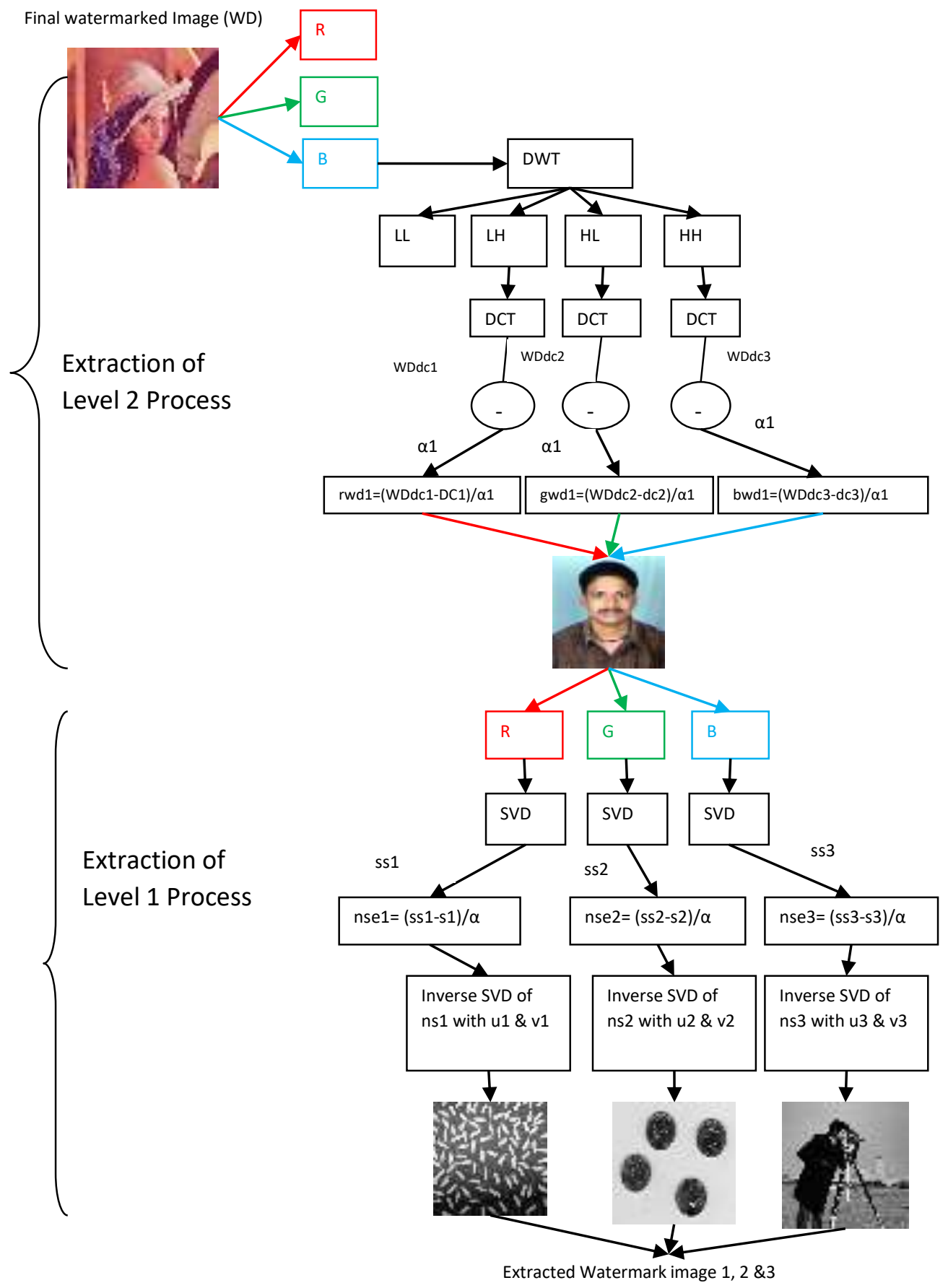


Fig. 3. Flow chart of watermark Extraction process

\section{EXPERIMENTAL RESULTS}

This section is given to experimental results based on proposed method. In this paper four different color host (cover) images and six different gray level watermark images. The size of cover images $512 * 512 * 3,256 * 256 * 3$ and watermarks are $256 * 256$. The simulation results were done by MATLAB 2009 software.

The quality of the proposed method is checked by using the parameters like peak-signal-noise ratio (PSNR), MSE (Mean Square Error) and RMSE (Root Mean Square Error).

The quality of the proposed method is shown as below figures from Fig 4-9.

\section{A. Peak Signal to Noise Ratio (PSNR)}

PSNR is one of the most useful parameter to measure the quality of the image. The PSNR is calculating the difference between original cover image (in1) and watermarked image (WD) [1, 3, and 5].

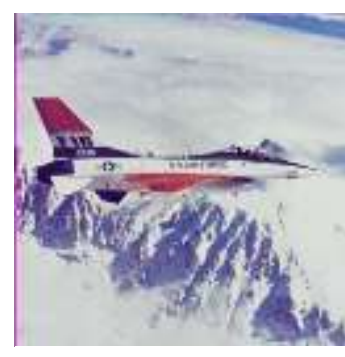

(a)

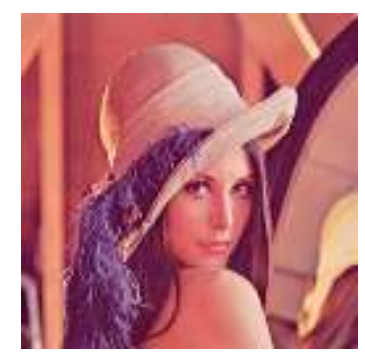

(b)

$$
\operatorname{PSNR}\left(d B^{\prime} s\right)=10 \log _{10}\left(\frac{L^{2}}{M S E}\right)
$$

Where $L$ is the Maximum gray level value i.e. 255

B. Mean Square Error (MSE)

$$
M S E=\frac{\sum_{X=0}^{X-1} \sum_{y=0}^{Y-1}(i n 1(x, y)-W D(x, y))^{2}}{X \times Y}
$$

\section{Root Mean Square Error (RMSE)}

The RMSE for the original cover image and watermarked image are defined as follows [7].

$$
R M S E=\sqrt{\frac{1}{X \times Y} \sum_{x=0}^{X-1 Y-1} \sum_{y=0}[i n 1(x, y)-W D(x, y)]^{2}}
$$

Fig.4. Existing method-(a) cover image in $1(512 * 512 * 3)$, (b) watermark images $(512 * 512 * 3)$, (c) watermarked image, (h) Extracted watermark image.

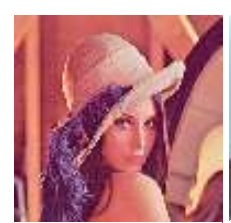

(a)

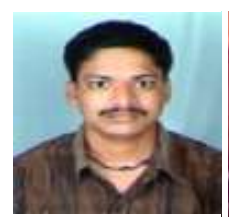

(f)

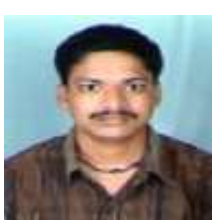

(b)

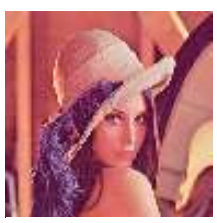

(g)

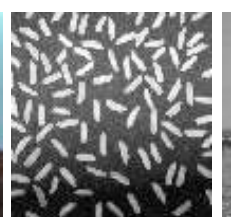

(c)

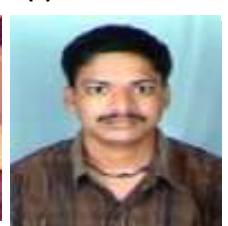

(h)

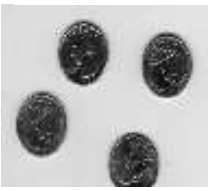

(k)

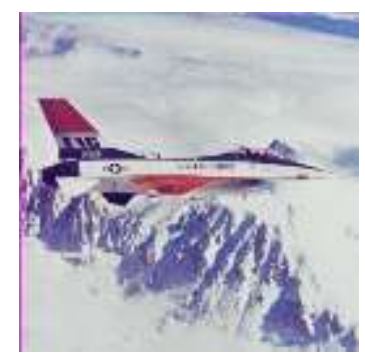

(c)

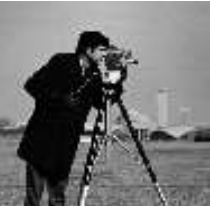

(d)

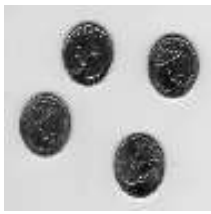

(e)

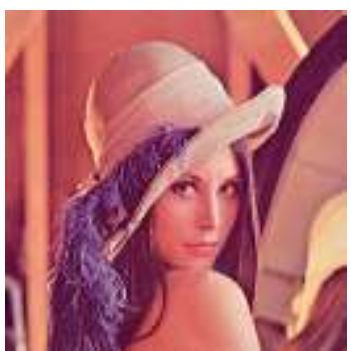

(d)

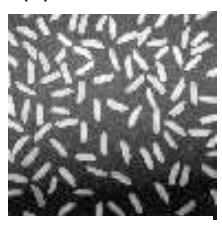

(i)

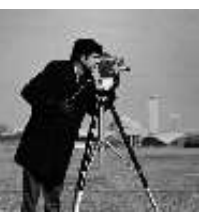

(j) 
Fig.5. Proposed method-(a) cover image in1 $(512 * 512 * 3)$, (b) cover image in2(256*256*3), (c-e) watermark images (256*256), (f) initial watermarked image, (g) Final watermarked image, (h) Extracted initial watermarked image, (i-k) Extracted watermark images.

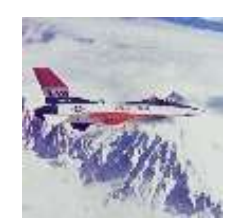

(a)

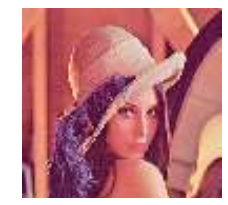

(f)

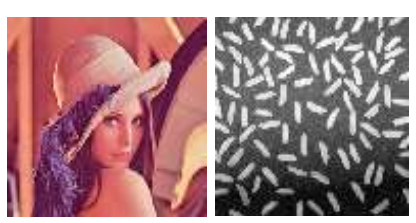

(c)

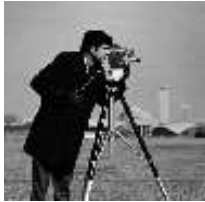

(d)

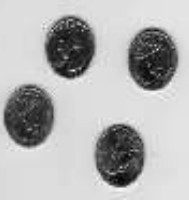

(e)

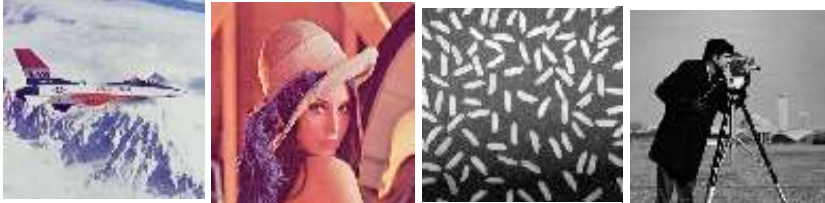

(g)

(i)

(j) (h)

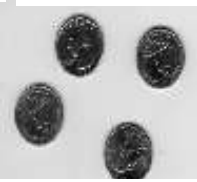

(k)

Fig.6. Proposed method-(a) cover image in1 (512*512*3), (b) cover image in2(256*256*3), (c-e) watermark images (256*256), (f) initial watermarked image, (g) Final watermarked image, (h) Extracted initial watermarked image, (i-k) Extracted watermark images.

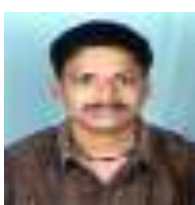

(a)

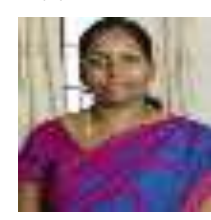

(f)

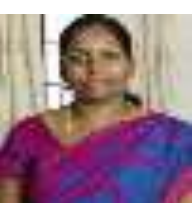

(b)

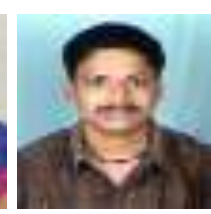

(g)

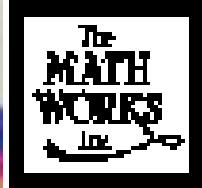

(c)

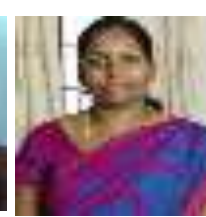

(h)

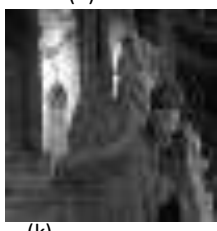

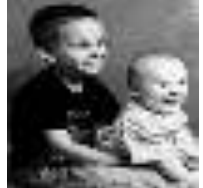

(d)

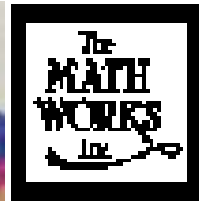

(i)

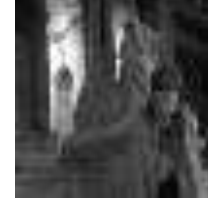

(e)

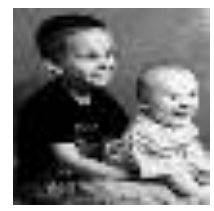

(j)

(k)

Fig.7. Proposed method-(a) cover image in $1(512 * 512 * 3)$, (b) cover image in2(256*256*3), (c-e) watermark images (256*256), (f) initial watermarked image, (g) Final watermarked image, (h) Extracted initial watermarked image, (i-k) Extracted watermark images. 
Helix Vol. 8(3):3478-3487

TABLE I. PERFORMANCE RESULTS IN TERMS OF PSNR, MSE \& RMSE

\begin{tabular}{|l|l|l|l|l|l|l|}
\hline Images & \multicolumn{2}{|l|}{ PSNR } & \multicolumn{2}{l|}{ MSE } & \multicolumn{2}{l|}{ RMSE } \\
\hline & Ref [6] & $\begin{array}{l}\text { Proposed } \\
\text { method }\end{array}$ & Ref [6] & $\begin{array}{l}\text { Proposed } \\
\text { method }\end{array}$ & Ref [6] & $\begin{array}{l}\text { Proposed } \\
\text { method }\end{array}$ \\
\hline man & 42.7142 & $\mathbf{5 7 . 1 8 7 2}$ & 3.4806 & $\mathbf{0 . 1 2 4 3}$ & 1.8656 & $\mathbf{0 . 3 5 2 5}$ \\
\hline Air & 45.1663 & $\mathbf{5 7 . 1 4 0 8}$ & 1.9790 & $\mathbf{0 . 1 2 5 6}$ & 1.4068 & $\mathbf{0 . 3 5 4 4}$ \\
\hline Lena & 44.6583 & $\mathbf{5 6 . 6 2 6 9}$ & 2.2246 & $\mathbf{0 . 1 4 1 4}$ & 1.4915 & $\mathbf{0 . 3 7 6}$ \\
\hline
\end{tabular}

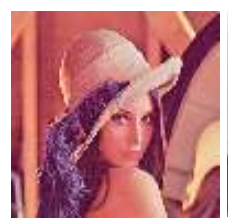

(a)

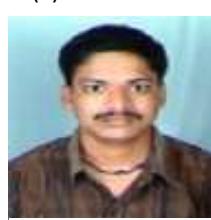

(f)

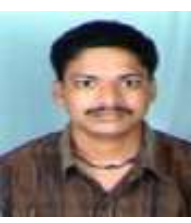

(b)

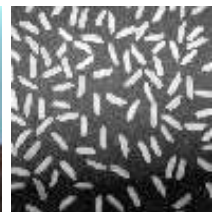

(c)

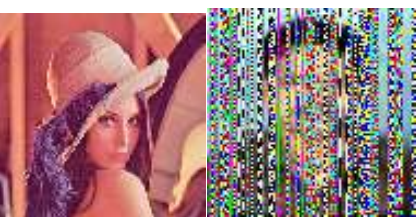

(g)

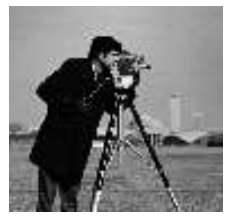

(d)

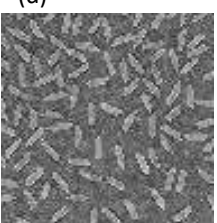

(i)

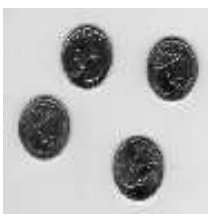

(e)

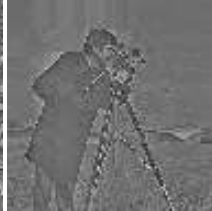

(j)

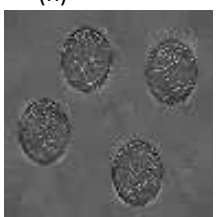

(k)

Fig. 8. Proposed method- salt and pepper noise 0.001 (a) cover image in $1(512 * 512 * 3)$, (b) cover image in2(256*256*3), (c-e) watermark images $(256 * 256)$, (f) initial watermarked image, (g) Final watermarked image, (h) Extracted initial watermarked image, (i-k) Extracted watermark images. 


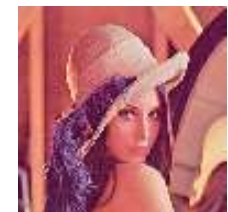

(a)

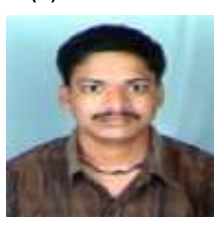

(f)

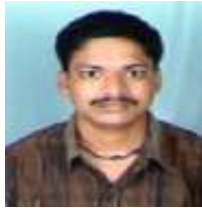

(b)

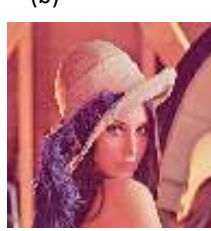

(g)

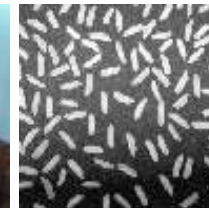

(c)

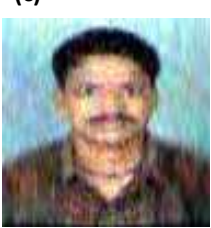

(h)

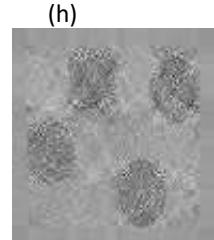

(k)

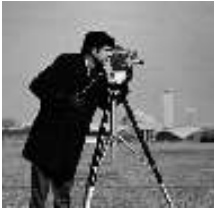

(d)

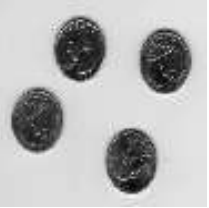

(e)

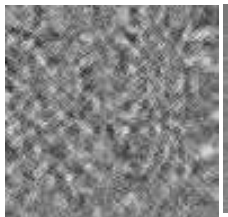

(i) (j)

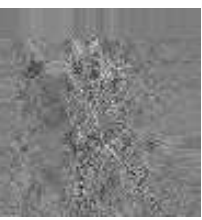

(j)

Fig.9. Proposed method- Brightness 3 (a) cover image in1 $(512 * 512 * 3)$, (b) cover image in2(256*256*3), (c-e) watermark images $\left(256^{*} 256\right)$, (f) initial watermarked image, (g) Final watermarked image, (h) Extracted initial watermarked image, (i-k) Extracted watermark images.

TABLE II. PERFORMANCE RESULTS IN TERMS OF PSNR, MSE \& RMSE WITH DIFFERENT NOISE ATTACKS (FIG 4)

\begin{tabular}{|c|c|c|c|c|c|}
\hline \multirow[t]{2}{*}{ Images } & \multirow[t]{2}{*}{ Noise / Attacks } & \multirow[t]{2}{*}{ Density } & \multicolumn{3}{|c|}{ Proposed method } \\
\hline & & & $\begin{array}{l}\text { PSNR } \\
\end{array}$ & MSE & RMSE \\
\hline & & 0.001 & 35.0196 & 20.4703 & 4.5244 \\
\hline & Salt\& pepper & 0.01 & 25.2262 & 195.1903 & 13.9711 \\
\hline & noise & 0.1 & 15.1742 & $1.98 \mathrm{E}+03$ & 44.446 \\
\hline & & 0.5 & 8.1895 & $9.87 \mathrm{E}+03$ & 99.3262 \\
\hline & Speckle noise & 0.3 & 11.7196 & $4.38 \mathrm{E}+03$ & 66.1545 \\
\hline \multirow[t]{9}{*}{ Lena } & & 0.5 & 10.0061 & $6.49 \mathrm{E}+03$ & 80.5814 \\
\hline & Gaussian noise & 0.01 & 20.2007 & 620.8786 & 24.9174 \\
\hline & & 0.05 & 13.846 & $2.68 \mathrm{E}+03$ & 51.7891 \\
\hline & & 0.1 & 11.5084 & $4.59 \mathrm{E}+03$ & 67.7833 \\
\hline & & 0.5 & 7.8532 & $1.07 \mathrm{E}+04$ & 103.248 \\
\hline & Brightness & 3 & 38.5222 & 9.1382 & 3.023 \\
\hline & & 5 & 34.1302 & 25.1224 & 5.0122 \\
\hline & & 7 & 31.2218 & 49.0793 & 7.0057 \\
\hline & & 10 & 28.1349 & 99.9066 & 9.9953 \\
\hline
\end{tabular}


Helix Vol. 8(3):3478-3487

TABLE III. PERFORMANCE RESULTS IN TERMS OF PSNR, MSE \& RMSE WITH DIFFERENT NOISE ATTACKS (FIG 5)

\begin{tabular}{|c|c|c|c|c|c|}
\hline \multirow[t]{2}{*}{ Images } & \multirow{2}{*}{$\begin{array}{l}\text { Noise / } \\
\text { Attacks }\end{array}$} & \multirow[t]{2}{*}{ Density } & \multicolumn{3}{|c|}{ Proposed method } \\
\hline & & & PSNR & MSE & RMSE \\
\hline \multirow{14}{*}{ air } & & 0.001 & 34.9371 & 20.8625 & 4.5676 \\
\hline & \multirow{3}{*}{$\begin{array}{l}\text { Salt\& } \\
\text { pepper } \\
\text { noise }\end{array}$} & 0.01 & 24.7727 & 216.677 & 14.72 \\
\hline & & 0.1 & 14.8489 & $2.13 \mathrm{E}+03$ & 46.1418 \\
\hline & & 0.5 & 7.8868 & $1.06 \mathrm{E}+04$ & 102.8491 \\
\hline & \multirow{2}{*}{$\begin{array}{l}\text { Speckle } \\
\text { noise }\end{array}$} & 0.3 & 9.9831 & $6.53 \mathrm{E}+03$ & 80.7949 \\
\hline & & 0.5 & 8.3704 & $9.46 \mathrm{E}+03$ & 97.2791 \\
\hline & \multirow{4}{*}{$\begin{array}{l}\text { Gaussian } \\
\text { noise }\end{array}$} & 0.01 & 20.1861 & 622.9685 & 24.9593 \\
\hline & & 0.05 & 14.1724 & $2.49 \mathrm{E}+03$ & 49.8796 \\
\hline & & 0.1 & 11.8094 & $4.29 \mathrm{E}+03$ & 65.4739 \\
\hline & & 0.5 & 7.8151 & $1.08 \mathrm{E}+04$ & 103.7015 \\
\hline & \multirow[t]{4}{*}{ Brightness } & 3 & 38.5276 & 9.1268 & 3.0211 \\
\hline & & 5 & 34.1294 & 25.1271 & 5.0127 \\
\hline & & 7 & 31.2176 & 49.1274 & 7.0091 \\
\hline & & 10 & 28.1253 & 100.1279 & 10.0064 \\
\hline
\end{tabular}

TABLE IV. PERFORMANCE RESUlTS IN TERMS OF PSNR, MSE \& RMSE WITH DIFFERENT NOISE ATTACKS (FIG 6)

\begin{tabular}{|c|c|c|c|c|c|}
\hline \multirow[t]{2}{*}{ Images } & \multirow[t]{2}{*}{ Noise / Attacks } & \multirow[t]{2}{*}{ Density } & \multicolumn{3}{|c|}{ Proposed method } \\
\hline & & & PSNR & MSE & RMSE \\
\hline & & 0.001 & 34.8482 & 21.2943 & 4.6146 \\
\hline & Salt\& pepper & 0.01 & 24.8292 & 213.8764 & 14.6245 \\
\hline & nolse & 0.1 & 14.8745 & $2.12 \mathrm{E}+03$ & 46.0062 \\
\hline & & 0.5 & 7.8709 & $1.06 \mathrm{E}+04$ & 103.0373 \\
\hline & Speckle noise & 0.3 & 11.5735 & $4.53 \mathrm{E}+03$ & 67.2771 \\
\hline & & 0.5 & 9.9103 & $6.64 \mathrm{E}+03$ & 81.4755 \\
\hline & Gaussian noise & 0.01 & 20.3608 & 598.4098 & 24.4624 \\
\hline & & 0.05 & 14.1283 & $2.51 \mathrm{E}+03$ & 50.133 \\
\hline
\end{tabular}


Helix Vol. 8(3):3478-3487

\begin{tabular}{lllll}
\hline \multirow{3}{*}{$\operatorname{man}$} & 0.1 & 11.7449 & $4.35 \mathrm{E}+03$ & 65.9622 \\
& 0.5 & 7.8009 & $1.08 \mathrm{E}+04$ & 103.8717 \\
& 3 & 38.6355 & 8.9028 & 2.9838 \\
& & & & \\
& & & & \\
& & & & \\
& & 34.2261 & 24.5736 & 4.9572 \\
& 7 & 31.3265 & 47.911 & 6.9218 \\
& 10 & 28.2653 & 96.9516 & 9.8464 \\
\hline
\end{tabular}

\section{CONCLUSION}

Watermarking is a process to protect the data \& provide maximum extent of security for unauthorized persons. In this paper, a novel algorithm is proposed for multiple hiding of image watermarking with DWT, DCT \& SVD. Initially the three watermark images are embedding into cover image to obtain watermarked image by using SVD with scaling factor. Again that watermarked image is embedding into another cover image to obtain final watermarked image using DWT and DCT with scaling factor. This method provides more security and imperceptibility. To extract original secret (watermark images)

\section{Acknowledgment}

The authors would like to thank to the QIS for their support to execute the project.

\section{References}

[1] Abdulmawla Najih, et.al., "Digital image watermarking based on angle

4 quantization in discrete contourlet transform", Journal of King Saud University, Elsevier, 2016, pp:1-7.

[2] Vivek Singh Verma, et.al. "Significant region based robust watermarking scheme in lifting wavelet", www.elsevier.com/locate/eswa , Elsevier, 2015,pp:81848195.

[3] Maruturi Haribabu, et. al., "A Secure \& Invisible Image Watermarking Scheme Based onWavelet Transform in HSI color space", Procedia Computer Science, Elsevier, 2016, pp:462-468.

[4] S. Anu, et.al., " Comparison of DCT, SVD and BFOA based

multimodal biometric watermarking systems", AEJ 2015,pp:1-14.

[5] Divjot Kaur Thind, et.al., "A Semi Blind DWT-SVD Video Watermarking", Elsivier, 2015,pp:1661-1667.

[6] Rita Choudhary, et.al., "A Robust image Watermarking Technique using 2-level Discrete Wavelet Transform (DWT)", IEEE, 2016. perform reverse process. By using this proposed method the PSNR values are $\mathbf{5 5}$ for different host images before the attacks. It is concluded that the embedding and extraction of the proposed method is robust and imperceptible ad also shows improvement over the existed method based on the PSNR, MSE \& RMSE values. Future work will focus on extending the algorithm using advanced transformation techniques to improve robustness.

[7] Maruturi Haribabu, et. al., "A New Approach of Medical Image Fusion

Using Discrete Wavelet Transform", ACEEE, IJSIP, 2013,pp:21-25. 